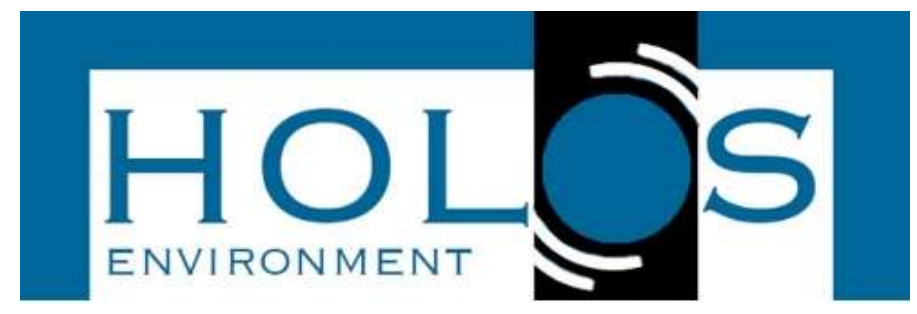

\title{
A GEOMETRIA DE POÇOS DE PETRÓLEO INTERFERE NA GERAÇÃo DE ÁGUA PRODUZIDA? - UM ESTUDO EM CAMPOS OFFSHORE DA BACIA POTIGUAR/BRASIL
}

\section{THE GEOMETRY OF PETROLEUM WELL INTERFERS IN THE GENERATION OF PRODUCED WATER? - A STUDY IN OFFSHORE OILFIELDS OF THE POTIGUAR BASIN/BRAZIL}

\author{
Marcus Vinicius Dantas de Assunção'; Mariana Rodrigues de Almeida²; \\ Marcela Marques Vieira²
}

Artigo recebido em: 05/04/2018 e aceito para publicação em: 17/09/2018.

DOI: http://dx.doi.org/10.14295/holos.v18i2.12283

Resumo: A geometria dos poços de petróleo pode ser decisiva quanto a geração de resíduos indesejáveis oriundos da produção de petróleo e gás. Um desses resíduos é a água produzida que tem aumentando seu volume nos campos petrolíferos e se tornado um grande desafio ambiental para os gestores desses campos. Diante deste cenário, o objetivo dessa pesquisa é analisar a influência da geometria dos poços petrolíferos na geração de água produzida de petróleo em campos offshore. Para tanto, a pesquisa foi desenvolvida em 8 campos de petróleo offshore, em fase de produção, na Bacia Potiguar, considerando, como amostra, os anos de 2014, 2015 e 2016. Formulou-se um modelo conceitual composto por três hipóteses, utilizando-se de regressões multivariadas. Os resultados foram apresentados em três etapas: (I) testes dos pressupostos, (II) apresentação e análise dos coeficientes da regressão e (III) análise das hipóteses de pesquisa. Os resultados mostram uma redução de $137,9 \%$ no volume de água produzida, em média, quando acrescido 1 poço vertical no projeto e um aumento de $13,8 \%$ para cada poço direcional incrementado no projeto. A idade do campo revelou-se a variável de maior influência na pesquisa, com um efeito médio de $23,7 \%$ sobre a produção de água.

Palavras-chave: Água Produzida. Petróleo. Regressão Multivariada. Geometria de Poços.

Abstract: The geometry of the oil wells can be decisive for the generation of undesirable residues from the production of oil and gas. One such waste is produced water that has increased its volume in the oil fields and has become a major environmental challenge for the managers of these fields. In view of this scenario, the objective of this research is to analyze the influence of oil well geometry on the generation of oil produced water in offshore fields. To do so, the research was developed in 8 offshore oil fields, in production phase, in the Potiguar basin, considering, as a sample, the years 2014, 2015 and 2016. A conceptual model was made up of three hypotheses, multivariate regressions. The results were presented in three stages: (I) assumptions tests, (II) presentation and analysis of regression coefficients and (III) analysis of the research hypotheses. The results show a $137.9 \%$ reduction in the volume of water produced, on average, when 1 vertical well was added to the project and a $13.8 \%$ increase for each incremental directional well in the project. The field age was the most influential variable in the research, with an average effect of $23.7 \%$ on the water produced.

\footnotetext{
${ }^{1}$ Instituto Federal de Educação, Ciência e Tecnologia do Rio Grande do Norte. Email: (marcus.assuncao@ifrn.edu.br)

2 Universidade Federal do Rio Grande do Norte (UFRN). E-mails: (almeidamariana@yahoo.com, marcela@geologia.ufrn.br)
} 
Palavras-chave: Water Produced. Oil. Multivariate Regression. Well Geometry.

\section{INTRODUÇÃO}

Com a alta demanda por petróleo, consequentemente, aumentou-se a quantidade de água produzida no processo de exploração/produção de óleo e gás, o que requer uma severa intervenção de práticas sustentáveis a fim de se evitarem danos ao meio ambiente. A origem da água produzida está relacionada às condições ambientais existentes durante a formação do óleo. Um ambiente geológico que tenha havido uma intensa deposição de matéria orgânica, associada com posterior soterramento e condições físico-químicas específicas tende a reunir os condicionantes necessários para o surgimento de petróleo nas rochas matrizes (ASSSUNÇÃO, VIEIRA \& ALMEIDA, 2018).

Quando esse óleo é extraído, por meio dos poços, seja em campos onshore ou offshore, produz resíduos aquosos ou efluentes denominadas águas residuais de campos petrolíferos ou água ou água de produção (AP), que é responsável pela maior quantidade de resíduos relativos à exploração de petróleo bruto (STEPHERSON, 1992; MONDAL \& WICKRAMASINGHE, 2008). A partir de uma produção excessiva a AP cria-se um problema sério em campos de petróleo, sobretudo, se forem maduros (FIGUEREDO et al., 2014).

A AP é uma parte indissociável no processo de recuperação de hidrocarbonetos. À medida que os campos envelhecem, estes tendem a produzir quantidades crescentes de água (KHATIB \& VERBEEK, 2003), podendo alcançar valores próximos de $100 \%$ da produção do poço ao final de sua vida produtiva. Os tipos de poços podem ser determinantes para rendimentos do campo petrolífero.

Além do fator idade, os tipos de poços de petróleo podem interferir na quantidade de AP que um campo venha a gerar. Os poços de petróleo têm múltiplos objetivos, dentre os quais destacam-se o fomento às informações sobre as formações geológicas e dados geofísicos (KAISER, 2007) que contribuem para o descobrimento de jazidas de petróleo e/ou gás e, consequentemente, iniciar a etapa de produção ou optar pelo abandono do poço.

Para que um poço seja construído é necessário que se tenha um projeto, cuja etapa inicial parte do estudo de área em que será perfurado, na qual analisa-se o cenário 
geológico e fazendo-se estudos dos poços previamente perfurados na região (ROCHA \& AZEVEDO, 2009).

A partir das informações colhidas, sobretudo acerca da geologia marinha, do conhecimento da profundidade do ponto alvo e da geometria esperada para o reservatório (ROCHA \& AZEVEDO, 2009), pode-se definir a trajetória para que o poço alcance o maior potencial de zona produtora. No que tange a geometria, os poços classificam-se em verticais, horizontais e direcionais.

Os verticais são poços cujo ponto do reservatório a ser atingido está na mesma linha vertical da sonda de perfuração (GABBAY et al., 2016), cujas principais características são: zero grau ou apenas poucos graus da linha vertical. A geometria mais utilizada na perfuração nos campos de petróleo é a vertical, uma vez que simplifica o processo de completação e avaliação do reservatório (ANP, 2017). Portanto, a partir dos estudos de Assunção, Vieira \& Almeida (2018), considera-se que nos campos offshore - campos situados no mar - haja maior eficiência utilizando-se os poços verticais, insurge-se a primeira hipótese de pesquisa - $\mathbf{H}_{\mathbf{1}}$ - Há influência negativa dos poços verticais na geração de AP em campos offshore.

Apesar de ser largamente requisitado nos campos de petróleo, os poços verticais apresentam limitações importantes, quais sejam: maior queda maior de pressão nas regiões próximas do poço em comparação com os poços horizontais; área de drenagem reduzida, especialmente em formações apertadas conhecidas como de baixa permeabilidade; pode ser necessário grande quantidade de poços para desenvolver um campo (ELYASI, 2016).

Os poços direcionais, diferentemente dos poços verticais, são perfurados em ângulos de 45 a 60 graus ou mais, a partir da linha vertical real, cujo objetivo está afastado da linha vertical atinge o topo do poço. O ângulo de perfuração da planta, define o afastamento do poço direcional, que permite classificá-los em convencionais, de grande afastamento e de afastamento severo (LAIK, 2018).

Os poços direcionais são utilizados frequentemente como poços de plataforma offshore para 'atingir' distâncias maiores a partir da plataforma e podem percorrer diversos estilos de trajetória a fim de atingirem seus objetivos. Em virtude de seus multiobjetivos operacionais, elevando-se, contudo, os custos intrínsecos agregados, propõe-se a segunda 
hipótese de pesquisa: $\mathbf{H}_{\mathbf{2}}$ - Há influência positiva dos poços direcionais na geração de AP em campos offshore.

Um tipo particular de poço direcional é o poço horizontal, cujo objetivo é proporcionar aumento de produtividade e de recuperação final de hidrocarbonetos. De acordo com Prado (2003), há duas categorias de aplicações de poços horizontais: resolução de problemas decorrentes das características de fluxo (escoamento) de fluidos e resolução de problemas decorrentes das heterogeneidades do reservatório. As duas aplicações podem estar presentes no mesmo sistema.

De acordo com Dehua, Guowei, Jing \& Wenying (2011), os poços horizontais possuem um segmento reto que é perfurado horizontalmente dentro da formação produtora, ampliando a sua área de drenagem no reservatório. São poços de longo alcance (extended reach wells) em que o objetivo está distante horizontalmente da sua locação na superfície $(>10 \mathrm{~km})$.

Esses poços, segundo Chaperon (1986) e Mukherjee \& Economides (1991) podem produzir até três vezes mais que os poços verticais, no entanto reúnem mais riscos no seu funcionamento e possui custos mais elevados que os poços verticais. Dada a eficiência desse tipo de poço, mas permeada por diversos riscos inerentes à operação, pode-se apresentar a terceira hipótese de pesquisa: $\quad \mathbf{H}_{3}$ - Há influência positiva dos poços horizontais na geração de água produzida em campos offshore.

Estudos anteriores de Chaperon (1986) compararam os poços verticais quanto à proximidade e interferência de cones críticos. $O$ resultado da comparação entre os dois tipos de poços mostra que um cone crítico se aproxima do poço horizontal mais do nos poços verticais. Além disso, os poços horizontais geralmente permitem maiores taxas críticas do que os poços verticais, porém essa vantagem diminui com o aumento da anisotropia.

Estudos relativos aos poços horizontais, Babu \& Odeh (1989) apresentaram uma equação para calcular o fluxo do estado pseudoestructural para um poço horizontal, similar a conhecida equação de produtividade para um poço vertical. A equação proposta objetiva estudar os efeitos na boa produtividade do comprimento do poço, localização e grau de penetração; permeabilidade vertical e horizontal; e a dimensão horizontal do volume de drenagem. 
Já Mukherjee \& Economides (1991) apresentaram critérios de triagem para poços verticais e horizontais com ou sem fraturas induzidas, bem como um procedimento para calcular o número ótimo de fraturas transversais ortogonais em poços horizontais.

Xu et al., (2014) desenvolveram um modelo de faturamento hidráulico para poços verticais com baixa permeabilidade e formações de óleo apertado quando a permeabilidade do reservatório é inferior a $0.1 \mathrm{mD}$. A análise de produtividade concluiu que quanto maior o gradiente de pressão limiar, menor a produtividade do poço de petróleo e quanto maior a sensibilidade ao estresse, menor a produtividade, além disso, a produtividade inicial do poço de petróleo aumenta com o aumento do comprimento da fratura, mas com taxa de aumento inferior.

Evoluindo os estudos anteriores, Assunção, Vieira \& Almeida (2018) estudaram os campos da Bacia Potiguar com foco nas instalações onshore. Os resultados autorizam afirmar que as variáveis independentes (idade do campo, grau API, tipo de campo, produção de óleo, e gás) interferem no volume de AP, apresentando-se como fatores determinantes para uma eficiente gestão deste resíduo nas bacias petrolíferas.

Diante do contexto apresentado, o presente trabalho tem como objetivo analisar a influência dos tipos de poços petrolíferos na geração de AP de petróleo em campos offshore. Para tanto, a pesquisa foi desenvolvida em 8 campos de petróleo offshore em fase de produção na Bacia Potiguar, considerando os anos de 2014, 2015 e 2016.

Esse estudo implica numa contribuição a ser incluída na literatura especializada para a área de petróleo, uma vez que trata de variáveis ainda não utilizadas na relação direta com a AP associada ao petróleo.

O resultado da pesquisa fomentará decisões estratégicas na área de sustentabilidade ambiental dos campos de petróleo por parte dos órgãos reguladores, das empresas exploradoras dos campos, bem como, o próprio governo, por meio de políticas públicas.

O estudo é composto por quatro seções, sendo a primeira seção dedicada à introdução, com uma abordagem voltada para um maior detalhamento sobre AP e tipos de poços de petróleo. A segunda seção trata dos procedimentos metodológicos utilizados, bem como as ferramentas estatísticas. Enquanto que a terceira seção traz os resultados e discussões a partir das análises dos dados. Por fim, concluir-se-á o estudo mediante a 
obtenção de respostas para o objetivo proposto e confirmação ou não das hipóteses suscitadas.

\section{MATERIAIS E MÉTODOS}

O presente estudo utiliza métodos quantitativos para apresentar seus resultados. $\mathrm{O}$ trabalho versa sobre a influência dos tipos de poços de petróleo na geração de AP em campos offshore da Bacia Potiguar.

A Bacia Potiguar, objeto de estudo de desta pesquisa, detém 22,5\% dos campos de petróleo do Brasil e aproximadamente 6.000 poços em produção, considerando o ano de 2016. Dos seus 106 campos de petróleo (10 offshore e 96 onshore), nos anos de 2014, 2015 e 2016, estavam 80 em fase de produção, destes, apenas 8 são offshore. Como representa um número insuficiente para mensuração de um modelo discricionário, optouse por analisar os dados de 3 anos, o que totaliza uma amostra de 24 campos.

Os dados coletados, de origem secundária, estão disponíveis no sítio eletrônico da ANP (Agência Nacional de Petróleo, 2017) em duas bases de dados, quais sejam: área dos campos e produção dos campos. As duas bases de dados foram aglutinadas a fim de que todas as variáveis de estudo estivessem localizadas em uma base única, tomando-se por referência os anos de 2014; 2015 e 2016.

As variáveis escolhidas para serem utilizadas na modelagem foram classificadas em três classes: variável dependente, as de controle e as independentes, conforme apresentadas no Quadro 1.

Quadro 1 - Variáveis de estudo

\begin{tabular}{|l|c|l|}
\hline Variável & Tipo de variável & \multicolumn{1}{c|}{ Descrição } \\
\hline Água produzida & Dependente & $\begin{array}{l}\text { Quantidade em m³ de água produzida gerada } \\
\text { com a produção de petróleo. }\end{array}$ \\
\hline Poços verticais & Independente & $\begin{array}{l}\text { Zero grau ou apenas poucos graus da linha } \\
\text { vertical real, geometria mais utilizada nos } \\
\text { campos de petróleo. }\end{array}$ \\
\hline Poços horizontais & Independente & $\begin{array}{l}\text { São poços de longo alcance (extended reach } \\
\text { wells) em que o objetivo está afastado } \\
\text { horizontalmente da sua locação na superfície } \\
(>10 \mathrm{~km}) .\end{array}$ \\
\hline Poços direcionais & Independente & $\begin{array}{l}\text { São poços perfurados em ângulos de 45 a 60 } \\
\text { graus ou mais, a partir da linha vertical real, }\end{array}$ \\
\hline
\end{tabular}




\begin{tabular}{|l|l|l|}
\hline & & $\begin{array}{l}\text { cujo objetivo está afastado da linha vertical } \\
\text { que passa pelo topo do poço. }\end{array}$ \\
\hline Grau API & Controle & $\begin{array}{l}\text { Escala utilizada para medir a densidade do } \\
\text { petróleo e seus derivados. }\end{array}$ \\
\hline Idade do campo & Controle & $\begin{array}{l}\text { Tempo de operação do campo, desde o início } \\
\text { da produção. }\end{array}$ \\
\hline
\end{tabular}

As variáveis independentes (Poços verticais, Poços horizontais, Poços direcionais) são utilizadas para se determinar o grau de explicação em relação a variável dependente, ou seja, servirão para mensurar a influência destas sobre a variável (água produzida), enquanto que as variáveis (Grau API e Idade do campo) servirão como variáveis de controle, as quais foram testadas em ambiente onshore por Assunção; Vieira \& Almeida (2018).

No intuito de mensurar a influência das variáveis independentes sobre o a geração de $A P$, utilizou-se a regressão múltipla, como técnica estatística multivariada. O " $X$ " é uma matriz de variáveis explicativas que buscam uma relação de causalidade com a variável Y e compõem as variáveis independentes do modelo: Poços verticais, Poços horizontais, Poços direcionais. Enquanto que o "W" corresponde a uma matriz de variáveis de controle (Grau API, Idade do campo). Essas relações estão representadas na Equação 1.

$$
\ln Y(A P)=\beta_{0}+\beta_{1}{ }^{*} X+\beta_{2}{ }^{*} W+\varepsilon
$$

$A P=$ água produzida; $\beta_{0}=$ constante do modelo; $\beta_{1}=$ Coeficientes; $\beta_{2}=$ Coeficientes.

$\beta$ o representa a constante do modelo; o $\varepsilon$, os resíduos; enquanto $\circ \beta$ significa o vetor de parâmetro de explicação para as matrizes $X$ e W. Se não forem considerados os sinais dos vetores de explicação, espera-se um modelo como o proposto na Equação 2.

In $Y($ Água produzida $)=\beta 0+\beta 1^{*}$ Poços Verticais $+\beta 2^{*}$ Poços horizontais $+\beta 3^{*}$ Poços

$$
\text { direcionais }+\beta 4^{*} \text { Idade do campo }+\beta 5^{\star} \text { Grau API }+\varepsilon
$$

$\mathrm{AP}=$ água produzida; $\beta_{0}=$ constante do modelo; $\beta_{1}=$ Coeficiente da variável poços verticais; $\beta_{2}=$ Coeficiente da variável poços horizontais; $\beta_{3}=$ Coeficiente da variável poços direcionais; $\beta_{4}=$ Coeficiente da variável idade do campo; $\beta_{5}=$ coeficiente da variável grau $\mathrm{API} ; \varepsilon=$ resíduos. 
O modelo multivariado poderá buscará demonstrar a relação da variável AP com todas as variáveis independentes e de controle, em um ambiente de simulação, com o objetivo de responder às hipóteses de pesquisa. Portanto, as hipóteses serão validadas se o conjunto de dados estudados confirmarem a hipótese de causalidade da variável AP com as variáveis independentes (Poços verticais, Poços horizontais, Poços direcionais).

No intuito de se linearizarem os dados, considerando a busca pela homocedasticidade dos resíduos, à variável AP foi aplicado o logaritmo neperiano (In). As análises foram conduzidas por meio da aplicação da relação Fator = 100[exp ( $\beta$ ) - 1] estabelecida por Wooldridge (2010).

\subsection{Análise dos pressupostos}

Após verificar duas iterações, seguindo os pressupostos da regressão múltipla, excluíram-se do modelo duas variáveis por apresentarem baixa significância estatística ( $p_{\text {valor }}$ $>0,05)$, quais sejam: Poços horizontais e Grau API.

Para validarem as regressões quanto aos seus pressupostos, foram realizados os testes de VIF, Tolerance (Multicolinearidade), Durbin-Watson (Autocorrelação), Pesaran-Pesaran (Homocedasticidade) e o teste de normalidade (Kolmogorov Smirnov). Os resultados quanto à multicolinearidade, testada pelo VIF e Tolerance, permitiram verificar a sua ausência, ou seja, os parâmetros enquadrame dentro da normalidade.

Quanto ao teste de normalidade do KS-sample (Kolmogorov-Smirnov), os dados indicaram normalidade, uma vez que se aceitou a hipótese nula, com estatística do teste 0,137 e pvalor $>0,05$. A autocorrelação foi testada por Durbin-Watson, obtendo-se o valor 2,14, compreendido no intervalo que atesta a ausência de autocorrelação. A verificação da homocedasticidade pelo teste de Pesaran-pesaran, com $F=0,91$ e pvalor $>0,05$. Os resíduos são homocedásticos, implicam na aceitação da hipótese nula. Portanto a regressão atende aos pressupostos da regressão multivariada, o que traz parcimônia aos resultados desta pesquisa.

Os resultados serão apresentados em três etapas: etapa I - será aplicada uma regressão múltipla com o intuito de mensurar a causalidade da variável independente, ou de controle, com a variável PA (dependente); e, etapa II - serão analisadas as hipóteses de pesquisa a fim validá-las ou refutá-las. Os testes de hipóteses serão realizados por meio do teste $\mathrm{T}$ e o software utilizado na consecução desta pesquisa foi o pacote SPSS (Statistical Package for tem Social Sciences), versão 24. 


\section{RESULTADOS E DISCUSSÃO}

Os resultados para serem analisados foram subdivididos em duas seções: 3.1 regressão multivariada; 3.2 avaliação do teste de hipóteses. Adotou-se, como nível de significância para esta pesquisa, 95\%.

Os $\mathrm{R}^{2}$ ajustado, presente na Tabela 1 , apresenta um fator de explicação das variáveis independentes em relação à dependente que variam aproximadamente de 68,3\%. Quando analisado o Teste $F$, verificou-se que, como o p-valor $(0,000)$ é menor que 0,05 , rejeita-se a hipótese de que $\mathrm{R}^{2}$ é igual a zero, ou seja, as variáveis independentes exercem influência sobre a dependente e o modelo mostra-se significativo.

Tabela 1 - Regressão multivariada para avaliação dos campos de petróleo

\begin{tabular}{|c|c|c|c|}
\hline & Iteração 1 & Iteração 2 & Iteração 3 \\
\hline Constante & $\begin{array}{l}3,183 \\
\left(p_{\text {valor }}=\right. \\
0,606)\end{array}$ & $\begin{array}{l}3,484 \\
\left(p_{\text {valor }}=\right. \\
0,301)\end{array}$ & $\begin{array}{c}4,366 \\
\left(p_{\text {valor }}{ }^{*}=\right. \\
0,000)\end{array}$ \\
\hline $\begin{array}{c}\text { Poços } \\
\text { horizontais }\end{array}$ & $\begin{array}{l}0,136 \\
\text { (pvalor }= \\
0,953)\end{array}$ & - & - \\
\hline API & $\begin{array}{c}0,028 \\
{\left(p_{\text {valor }}^{*}=\right.}_{0,826)}\end{array}$ & $\begin{array}{l}0,022 \\
\left(p_{\text {valor }}=\right. \\
0,782)\end{array}$ & - \\
\hline $\begin{array}{l}\text { Poços } \\
\text { verticais }\end{array}$ & $\begin{array}{c}-1,408 \\
\left(p_{\text {valor }}{ }^{*}=\right. \\
0,005)\end{array}$ & $\begin{array}{c}-1,420 \\
\text { (pvalor }^{*}= \\
0,001)\end{array}$ & $\begin{array}{c}-1,379 \\
\left(p_{\text {valor }}{ }^{*}=\right. \\
0,001)\end{array}$ \\
\hline $\begin{array}{l}\text { Idade do } \\
\text { campo }\end{array}$ & $\begin{array}{c}0,247 \\
\left(p_{\text {valor }}^{*}=\right. \\
0,005)\end{array}$ & $\begin{array}{c}0,243 \\
\left(p_{\text {valor }}^{*}=\right. \\
0,000)\end{array}$ & $\begin{array}{c}0,237 \\
\left(p_{\text {valor }}{ }^{*}=\right. \\
0,000)\end{array}$ \\
\hline $\begin{array}{l}\text { Poços } \\
\text { direcionais }\end{array}$ & $\begin{array}{c}0,138 \\
\text { (pvalor }^{*}= \\
0,005)\end{array}$ & $\begin{array}{c}0,141 \\
\text { (pvalor }^{*}= \\
0,002)\end{array}$ & $\begin{array}{l}0,138 \\
\text { (pvalor }^{*}= \\
0,001)\end{array}$ \\
\hline $\mathrm{R}^{2}$ & 0,650 & 0,668 & 0,683 \\
\hline $\begin{array}{c}\text { F } \\
\text { Significância }\end{array}$ & $\begin{array}{c}9,527 \\
\left(p_{\text {valor }}{ }^{*}=\right. \\
0,000)\end{array}$ & $\begin{array}{c}12,567 \\
\text { (palor }^{*}= \\
0,000)\end{array}$ & $\begin{array}{c}17,538 \\
\left(p_{\text {valor }}{ }^{*}=\right. \\
0,000)\end{array}$ \\
\hline
\end{tabular}

Retomando a Equação (1) e de acordo com os sinais e coeficientes resultantes do modelo de regressão descrito na Tabela 1, a Equação (3) foi estimada: 
In (Água produzida $)=4,366+0,237^{*}$ Idade $-1,379^{*}$ Poços verticais $+0,138^{*} P o c ̧ o s$ direc $+\varepsilon$

A partir da Equação (3), depreende-se que a escolha do poço vertical para um projeto petrolífero, representa, um efeito negativo no que concerne à geração de AP de petróleo, ou seja, para cada incremento de 1 poço vertical no projeto de poços offshore, há uma diminuição, em média, de 137,9\% de AP, o que podem significar ganhos significativos ao meio ambiente, o que diverge dos resultados apontados por Assunção, Vieira \& Almeida (2018), cujos resultados apresentaram uma variação positiva de $0,5 \%$, mas para campos onshore.

A variável "poços direcionais" apresentou um efeito positivo sobre a variável dependente com um impacto, em média, de aproximadamente $13,8 \%$ de aumento de AP a cada 1 poço direcional em produção offshore, convergindo com os resultados de Assunção, Vieira \& Almeida (2018) que demonstraram um incremento de 1,9\% para esse tipo de poço em campos onshore.

A variável idade mostrou-se fundamental na geração de AP para os campos offshore da Bacia Potiguar. Esta afirmação está em consonância com o estudo de Khatib \& Verbeek (2003), o qual consideram o aumento da idade dos campos fator determinante para o aumento de AP. Para cada variação de 1 ano de produção, em média, há um aumento na geração de AP de $23,7 \%$ considerando os poços offshore da Bacia Potiguar. Esta variável apresentou ainda o maior coeficiente padronizado Beta, o que significa ser a variável de maior impacto sobre a variável dependente AP.

Cabe ressaltar que a variável "poços horizontais" não se mostrou significativa para a presente análise. Isso ocorreu pelo fato de apenas três campos de petróleo offshore da Bacia Potiguar apresentarem poços horizontais, tornando-os outliers da pesquisa para essa variável. Além disso, por serem os poços horizontais do tipo direcional, o que ensejou uma multicolinearidade entre essas duas variáveis. Outra variável que não resultou em significância estatística foi API. Realizaram-se testes do tipo "T" com níveis de significância de $95 \%$ e 99\%, cujos resultados auferidos estão descritos na Tabela 2. 
Tabela 2 - Testes T para as Hipóteses da pesquisa

\begin{tabular}{|c|c|c|c|}
\hline \multirow{2}{*}{\multicolumn{2}{|c|}{ Hipótese $1\left(\mathbf{H}_{1}\right)$}} & \multirow{5}{*}{$\begin{array}{l}-4,007 \\
0,001^{*}\end{array}$} & Status \\
\hline & & & \multirow{4}{*}{ Validada } \\
\hline \multirow{2}{*}{$\begin{array}{l}\text { Poços } \\
\text { Verticais }\end{array}$} & Teste $t$ & & \\
\hline & $P_{\text {valor }}$ & & \\
\hline \multicolumn{2}{|c|}{ Hipótese $2\left(\mathbf{H}_{2}\right)$} & & \\
\hline \multirow{2}{*}{$\begin{array}{l}\text { Poços } \\
\text { direcionais }\end{array}$} & Teste $t$ & \multirow{3}{*}{$\begin{array}{l}3,755 \\
0,001^{*}\end{array}$} & \multirow[t]{3}{*}{ Validada } \\
\hline & $P_{\text {valor }}$ & & \\
\hline \multicolumn{2}{|c|}{ Hipótese $3\left(\mathbf{H}_{3}\right)$} & & \\
\hline \multirow{2}{*}{$\begin{array}{l}\text { Poços } \\
\text { horizontais }\end{array}$} & Teste $\mathrm{t}$ & \multirow{2}{*}{$\begin{array}{l}0,060 \\
0,953 \\
\end{array}$} & \multirow[t]{2}{*}{ Refutada } \\
\hline & $P_{\text {valor }}$ & & \\
\hline
\end{tabular}

Fonte: Dados da pesquisa (2018)

A Hipótese 1 da pesquisa foi validada, ou seja, pode-se constatar que o volume de AP diminui à medida que o campo de petróleo adota os poços verticais como recurso tecnológico estratégico para a produção de petróleo.

A Hipótese $2\left(\mathbf{H}_{2}\right)$ versa sobre a influência dos poços direcionais no volume de AP. Como a hipótese alternativa foi aceita, isso significa que a Hipótese 2 pode ser validada, uma vez que apresentou uma causalidade positiva. Atestou-se que o campo de petróleo que adota poços direcionais aumenta a geração de AP.

A Hipótese $3\left(\mathrm{H}_{3}\right)$ trata da influência poços horizontais de petróleo em relação à produção de água. Diferentemente das Hipóteses $\mathrm{H}_{1}$ e $\mathrm{H}_{2}$, na terceira Hipótese, a hipótese alternativa foi rejeitada, uma vez que não houve significância estatística para essa variável em nenhum dos anos estudados. Portanto, refuta-se a variável $\mathrm{H}_{3}$.

\section{CONCLUSÕES}

O objetivo deste trabalho consistiu em analisar a influência dos tipos de poços petrolíferos na geração de AP de petróleo a partir dos dados de 8 campos de petróleo offshore em fase de produção na Bacia Potiguar, considerando, como amostra, os anos de 2014; 2015 e 2016 para cada campo. Para tanto, desenvolveram-se três hipóteses vinculadas as variáveis independentes do estudo (Poços verticais, Poços direcionais e Poços horizontais).

As hipóteses formuladas para esta pesquisa foram testadas com significâncias de 95\% e 99\%, por meio dos testes T (regressões múltiplas). A Hipótese 1 foi confirmada com 
99\% de significância estatística, podendo-se afirmar, portanto, que há causalidade negativa entre a utilização de poços verticais e a AP de petróleo para os campos petrolíferos da Bacia Potiguar.

A hipótese 2 foi confirmada, com 99\% de significância, o que permite afirmar que há causalidade positiva entre a variável Poços direcionais e AP, ou seja, quanto maior for a quantidade de poços direcionais, maior será a AP.

A variável Poços horizontais foi refutada por meio da Hipótese 3, não se mostrando significativa. Ressalta-se como importante que os poços horizontais são pouco presentes nos campos de petróleo da Bacia Potiguar, o que contribuiu para uma resposta inconclusiva para essa variável.

Apesar de não ser uma variável independente, mas de controle para essa pesquisa, a idade do campo de petróleo denota ser fundamental para a AP apresentando um efeito positivo.

Portanto, os resultados matemáticos autorizam afirmar que as variáveis independentes Poços verticais e Poços direcionais interferem no volume de AP, apresentando-se como fatores determinantes para uma eficiente gestão da água residual nos campos petrolíferos da Bacia Potiguar, podendo prejudicar a sustentabilidade ambiental do campo offshore se suas quantidades e geometrias não forem adequadas a determinadas regiões exploradas.

Sugere-se, como pesquisas futuras, a aplicação da metodologia adotada, utilizandose os dados de todos os campos de petróleo offshore do Brasil com o intuito de melhor se aferirem os resultados nas demais bacias petrolíferas do Brasil. A pesquisa poderá ainda ser ampliada a partir da inclusão de outras variáveis, sobretudo se possuírem um viés econômico.

\section{REFERÊNCIAS}

Agência nacional de Petróleo (Brasil). Dados de exploração e produção - Consulta de área dos campos. Disponível em: http://www.anp.gov.br/wwwanp/exploracao-e-producao-de-oleo-egas/gestao-de-contratos-de-e-p/dados-de-e-p. Acesso em: 12 mai. 2017.

Agência nacional de Petróleo (Brasil). Dados de exploração e produção - Consulta de produção mensal de hidrocarbonetos. Disponível em: http://www.anp.gov.br/wwwanp/exploracao-eproducao-de-oleo-e-gas/gestao-de-contratos-de-e-p/dados-de-e-p. Acesso em: 12 mai. 2017.

ASSUNÇÃO, M. V. D., VIEIRA, M. M., ALMEIDA, M. R. The influence of oil well geometries on the produced water generation in onshore oilfields from the Potiguar Basin/Brazil. Acta Scientiarum Technology. v. 40, p. 1-7, 2018. 
ASSUNÇÃO, M. V. D., VIEIRA, M. M., ALMEIDA, M. R. Fatores influenciadores na produção indesejada da água produzida de petróleo: um estudo na bacia Potiguar/Brasil. Holos, v.42, p. 142-160, 2018.

BABU, D. K., ODEH, A. S. Productivity of a Horizontal Well (includes associated papers 20306, 20307, 20394, 20403, 20799, 21307, 21610, 21611, 21623, 21624, 25295, 25408, 26262, 26281, 31025, and 31035). SPE Reservoir Engineering, v. 4, n. 04, p. 417-421, 1989.

CHAPERON, I. Theoretical study of coning toward horizontal and vertical wells in anisotropic formations: subcritical and critical rates. In: SPE Annual Technical Conference and Exhibition. Society of Petroleum Engineers, p. 1-12, 1986.

DEHUA, L., GUOWEI, Z., JING, S., WENYING, L. Optimization design of horizontal wells development in low permeability gas reservoir in Su 53-4 Well area. Procedia Engineering. v. 18, p. 398-404, 2011.

ELYASI, S. Assessment and evaluation of degree of multilateral well's performance for determination of their role in oil recovery at a fractured reservoir in Iran. Egyptian Journal of Petroleum, v. 25, n. 1, p. 1-14, 2016.

FIGUEREDO, K. S. L., MARTíNEZ-HUITLE, Carlos. A., TEIXEIRA, A. B. R., PINHO, A. L. S., VIVACQUA, C. A., SILVA, D. R. Study of produced water using hydrochemistry and multivariate statistics in different production zones of mature fields in the Potiguar Basin-Brazil. Journal of Petroleum Science and Engineering, v. 116, p. 109-114, 2014.

GABBAY, M. S., DUTRA JUNIOR, T. V., MATA, W., RODRIGUES, M. A. F., LIRA, G. A. R. Análise crítica dos fatores que impactam o custo/metro dos poços de petróleo de um Campo de Produção localizado no Brasil. Petro \& Química, v. 1, p. 64-70, 2016.

KAISER, M. J. A survey of drilling cost and complexity estimation models. International Journal of Petroleum Science and Technology, v. 1, n. 1, p. 1-22, 2007.

KHATIB, Z., VERBEEK, P. Water to value-produced water management for sustainable field development of mature and green fields. Journal of Petroleum Technology, v. 55, n. 01, p. 2628, 2003.

\section{LAIK, S. Offshore Petroleum Drilling and Production. CRC Press, 2018.}

MONDAL, S.; WICKRAMASINGHE, S. Ranil. Produced water treatment by nanofiltration and reverse osmosis membranes. Journal of Membrane Science, v. 322, n. 1, p. 162-170, 2008.

MUKHERJEE, H., ECONOMIDES, M. J. A parametric comparison of horizontal and vertical well performance. SPE Formation Evaluation, v. 6, n. 02, p. 209-216, 1991.

ROCHA, L. A. S., AZEVEDO, C. T. de. Projetos de poços de petróleo-geopressões e assentamento de colunas de revestimentos. 2. ed. Rio de Janeiro: Interciência, 2009.

STEPHENSON, M. T. Components of produced water: a compilation of industry studies. Journal of Petroleum Technology, v. 44, n. 05, p. 548-603, 1992.

PRADO, R. P. S. Comparação entre estratégias de produção utilizando poços verticais e horizontais. 2003. 135f.. Dissertação (Ciências e Engenharia de Petróleo) - Universidade Estadual de Campinas, Campinas.WOOLRIDGE, J. M. Introdução à econometria: Uma abordagem 
moderna; tradução José Antônio Ferreira; revisão técnica Galo Carlos Lopez Noriega. São Paulo: Cengage Learning, 2010.

XU, Y. G., LI, X. W., LIAO, R. Q., XU, D. J. Productivity Analysis of Fractured Well in Tight Oil Reservoir. In: Advanced Materials Research. Trans Tech Publications, 2014. p. 712-715. 